\title{
The geography of prostate cancer incidence in Norway: Are the patterns real?
}

\author{
Asbjørn Aase $^{1}$ and Graham Bentham ${ }^{2}$ \\ ${ }^{I}$ Department of Geography, Norwegian University of Science and Technology, N-7034 Trondheim, Norway \\ ${ }^{2}$ Centre for Environmental Risk, School of Environmental Sciences, University of East Anglia, Norwich NR4 7TJ, England
}

\begin{abstract}
SUMMARY
The etiology of prostate cancer is to a large extent unknown. There are striking international variations in incidence, which may indicate that factors that show geographical variations can provide further clues about etiology. A problem with using incidence data for comparisons in time and space is that the number of cases reported may be affected by the intensity of diagnosing, since many of the cases are latent and asymptomatic. The purpose of this study is to adjust the observed pattern of prostate cancer in communes and counties of Norway for variations which may be due to diagnostic artefacts. It is assumed that a large proportion of local cancers may be an indication of more intensive diagnosing. Data of prostate cancer incidence for 1982-91 with tumours specified by degree of spread were provided by the Cancer Registry. A regression function relating total incidence to \% local tumours was used to predict the SIRs adjusted for variations in \% local tumours. The maps comparing incidence patterns before and after adjustement show that a large part of the significant deviations from the national mean persists, and that the pattern of negative deviations in the far north is even strengthened. A significant positive correlation between the ratio of incidence to mortality against \% local tumours is found, which supports the main hypothesis of the study.
\end{abstract}

\section{INTRODUCTION}

Prostate cancer is one of the most important cancers among men. In many western populations, such as Norway (Harvei et al. 1996), Sweden (Grönberg et al. 1996) and the United States (Boyle et al. 1995) it is the commonest recorded male cancer. However, the etiology of the disease is to a large extent unknown with only three patterns being well established: an increase with age, family history, and ethnic/geographical variations (Key 1995). Other factors which have been investigated are animal fat and dairy products consumption, vasectomy, sexual activitity and sexually aquired infection (Key 1995; Grönberg et al. 1996). Although these factors have been found to be statistically significant in some studies, the risk ratios are inconsistent and in most cases only slightly elevated. Some European studies show a positive association with social status, but other studies do not (Ross and Schottenfeld 1996). There are also inconsistencies with regard to rural/urban differences, with urban excesses in some countries and rural ones in others (Ross and Schottenfeld 1996).

International variations in incidence are very striking, with ratios in the magnitude of 1:100 between high and low incidence countries. The highest incidence rates are found among black people in the USA. Other high incidence populations are whites in North America, the Swiss and the Scandinavians. Particular- ly low incidence is found in some populations in China and other Asian countries (Boyle et al. 1995). The variations in mortality are mostly consistent with incidence patterns (Muir et al. 1991). Although it seems clear that genetic factors are important and may explain some of the geographical variations, these are so large that environmental factors must also be important. The fact that some Chinese and Japanese populations who have emigrated to Hawaii and California have higher incidences than is found in their areas of origin strengthens this assumption (Muir et al. 1991).

The existence of large differences in prostate cancer in different geographical areas could provide new clues about etiology. However, comparative studies of incidence of prostate cancer are, to a larger extent than with most other neoplasms, faced with difficulties of inaccuracy in reporting. A significant aspect of the disease is that many cases are asymptomatic and are only detected at autopsy, or coincidentally during investigations or treatment for non-malignant prostatic disorders, or by active screening by rectal examination or prostate specific antigen (PSA) testing. The large portion of asymptomatic cases creates difficulties for the use of prostate incidence data for epidemiological studies because the intensity of such activities obviously affects the number of cases which are detected. When studying secular trends the time series data may become biased by changes in diagnostic practice. For the same reason variations in incidence 
between countries, or between regions within a country, at a given time may also be affected.

One way to attempt to overcome these difficulties is to use data for mortality instead of incidence, since these are less likely to be affected by diagnostic bias than incidence (although the fact that a majority of prostate cancer cases occurs among older men may create diagnostic uncertainties). There are, however, good reasons for keeping incidence and mortality as separate categories. In order to measure changes in survival over time, for instance due to improvements in therapeutic methods, one must have consistent data on incidence as well as mortality (Helgesen et al. 1996). It is also important to be able to judge whether geographical variations in survival are due to differences in the measurement of incidence, or whether it reflects different therapeutic regimes or maybe different disease manifestations.

The other possibility is to make incidence data more robust to artefactual variation by using subcategories of diagnosis relating to degree of progression of the tumour. These categories are not included in the International Classification of Diseases (ICD), but are contained in some, but not all, cancer registries. Prostate cancers can be characterized by the stage of spread of the tumours, with localised tumours representing an earlier stage than those that have spread regionally or produced distant metastases. The assumption underlying the use of data on progression of the tumour for adjusting incidence rates, is that the more severe cases are likely to be detected under any diagnostic system, whereas the reporting of local tumours will tend to vary with the intensity of screening. On the other hand it can be argued that technically less sophisticated diagnosing of individual cases may lead to a misclassification as local instead of metastatic.

In the recent epidemiological literature on prostate cancer, a series of studies have been concerned with adjusting time series data for artefactual influences from changes in diagnostic practice (Grönberg et al. 1994; Gilliland et al. 1995; Jacobson et al. 1995). Less attention has been paid to the problem of whether geographical variations may be real or artefactual, although one study (Gilliland et al. 1996) has compared death risks for different ethnic groups adjusting for stage at diagnosis as well as tumour differentiation. The geographical variations in incidence might reflect variations in the completeness of registration as well as real differences. The purpose of this study is:

1. To describe geographical variations in the recorded incidence of prostate cancer in Norway.

2. To assess what part of the observed variation in incidence may be the result of artefacts of registration.

3. To adjust the observed patterns of incidence for artefacts to provide a firmer basis for further etiological investigation.

\section{DATA AND METHODS}

Data on incidence of prostate cancer by communes (439 units) and counties (fylker, 19 units) for the period 1982-91 were taken from a Norwegian Cancer Registry publication (Kreftregisteret 1993). Expected numbers of cases based on the age structure of the population and national age specific incidence rates were also abstracted. Dividing the observed number of cases by the expected then gave the indirectly standardised incidence ratio (SIR). Unpublished data on stage at diagnosis for each case (whether it was localised or whether it showed regional spread or distant metastases) was also supplied by the Norwegian Cancer registry for the same time period. It was therefore possible to calculate for each county the percentage of all cases where the tumours were localised. The small number of cases in many of the smaller communes meant that it would have been statistically inappropriate to undertake similar calculations for each commune. Instead, data for each commune was aggregated into 51 hospital catchment areas (Pedersen and Jørgensen 1991) and the percentage of localised tumours for these larger areas calculated. Individual communes were assigned the value of the hospital catchment area of which they were a part. As well as providing more stable values this approach also has the merit of basing the computation of values on the areal unit which is most likely to reflect possible variations in diagnostic practice.

The next step was to use regression analysis to examine whether incidence rates for prostate cancer were higher where the percentage of localised tumours was higher, as would be the case if intensive diagnostic activity led to the detection of a greater proportion of latent tumours. At the county level ordinary least square regression was used, with standardised prostate incidence ratio as the dependent variable and the percentage of localised tumours in the county as the explanatory variable. At the scale of communes, however, this approach would be statistically inappropriate because many small communes have a low number of cancer cases. Instead, Poisson regression analysis was used in which the dependent variable was the actual number of prostate cancer cases and the explanatory variable was the percentage of localised tumours in the hospital catchment area within which the commune was located. A model was fitted using a Poisson error distribution and a logarithmic link function with the logarithm of the expected number of cancer cases being fitted as an offset.

The regression models could then be used to predict the SIR that would be expected for each area from its percentage of localised tumours. Multiplying the expected number of cases by this value gave the number of cases that would be expected from its population age distribution and its percentage of localised tumours. Dividing the observed number of cases by 
this new expected value gave an SIR standardised for age and adjusted for variations in percentage of localised tumours.

Maps were produced by the Norwegian Social Science Data Service.

\section{GEOGRAPHICAL PATTERNS OF RECORDED PROSTATE CANCER INCIDENCE}

Fig. 1 shows a map of age adjusted incidence ratios of prostate cancer for the period 1982-91 for counties. Units showing significant positive or negative deviations ( 0.05 level, two-tailed test) from the national mean, are indicated. A latitudinal trend emerges, with lower incidence in the north than in the south, especially in the two northernmost counties Troms and Finnmark. In central and southern Norway the trend is less regular. There is a tendency, however, for higher incidence in the west than in the east.

The incidence pattern for communes gives a very fragmented picture. In fig. 2 we therefore show only units which deviate significantly from the national mean. The most prominent feature is the pattern of negative deviations in the three northernmost counties (especially in Finnmark in the far north), with 14 units showing significantly negative deviations and only one positive. In central and southern Norway the pattern is more fragmented and difficult to interpret. Small clusters of positive deviations appear in the south and west, and there are more negative than positive deviations in the south east.

\section{Incidence of prostate cancer 1982-91 Counties in Norway}

Observed/expected (Norway)

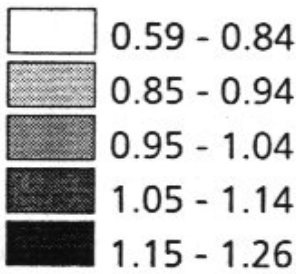

- significantly low

+ significantly high

Jata: Norwegian cancer registry

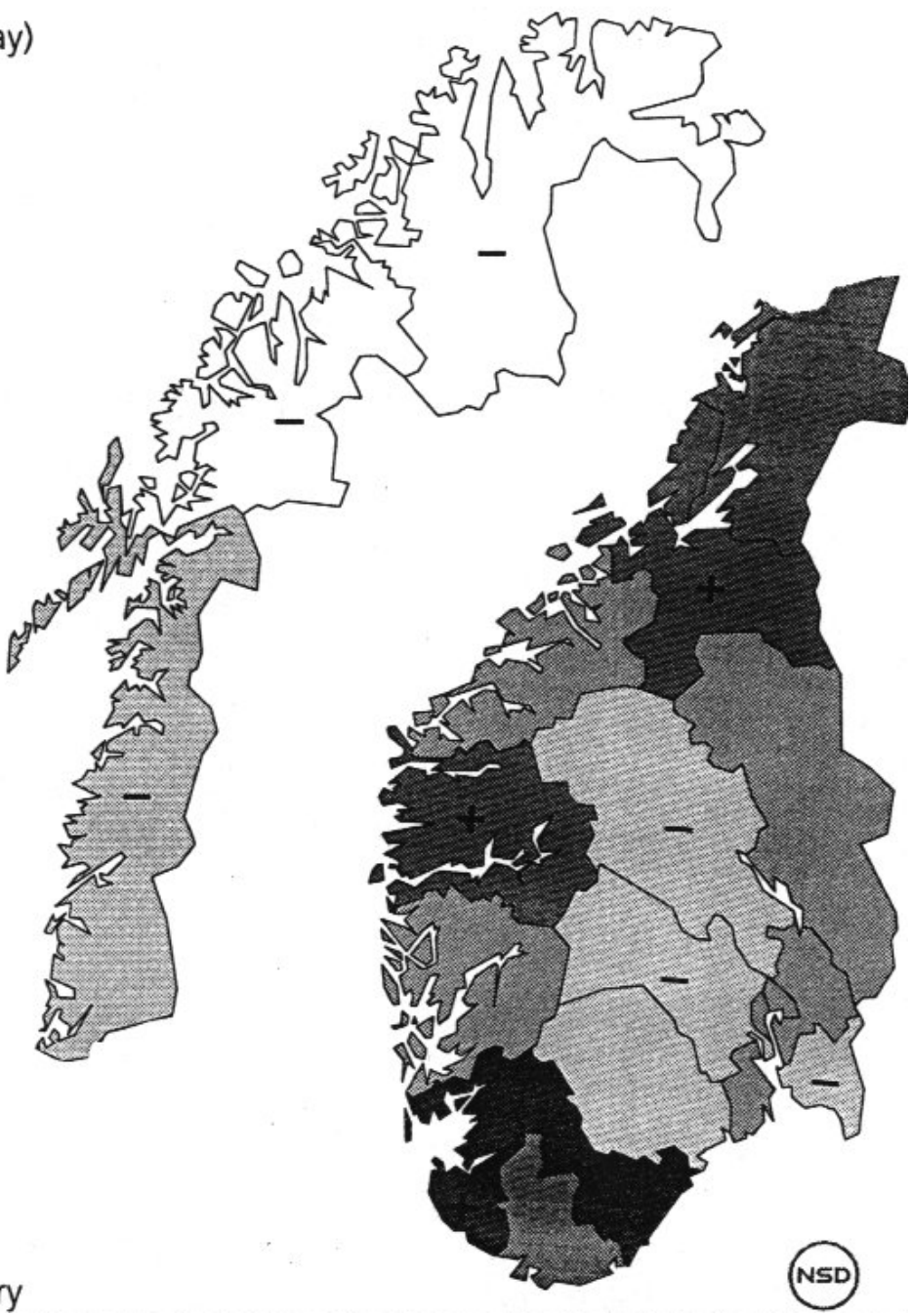

Figure 1. Incidence of prostate cancer in Norwegian counties, 1982-91. 


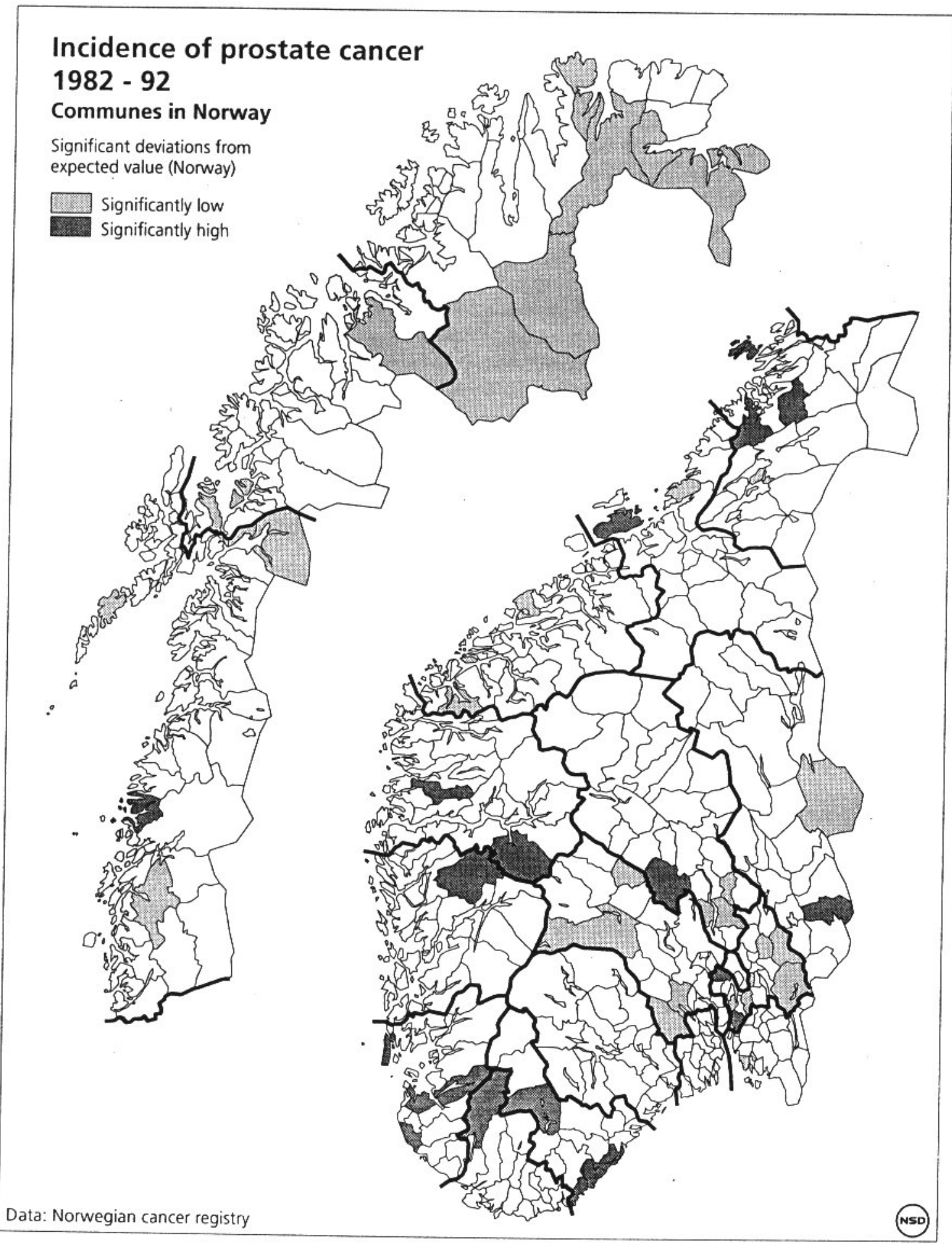

Figure 2. Incidence of prostate cancer in Norwegian communes, 1982-91.

\section{THE LATITUDINAL GRADIENT}

Using data on observed and expected number of cases for communes and then aggregating them into bands covering 1 degree of latitude it is possible to see whether the latitudinal trend for incidence shows a smooth trend from south to north (Table 1). If the gradient were the result of some physical factor varying latitudinally a regular gradient might be expected. The table shows substantial variations between similar latitudes rather than a smooth gradient. It therefore seems likely that the apparent association with latitude reflects the operation of some other factor which discriminates between the northernmost latitudes on the one hand and the rest of the country on the other. 
Table 1. Standardised incidence ratios (SIR) of prostate cancer 1982-91 by degrees of latitude.

\begin{tabular}{cc}
\hline Latitude & SIR 1982-91 \\
\hline 59 & 1.207 \\
60 & 0.978 \\
61 & 0.990 \\
62 & 1.016 \\
63 & 0.964 \\
64 & 1.063 \\
65 & 1.108 \\
66 & 0.895 \\
67 & 1.030 \\
68 & 1.013 \\
69 & 0.845 \\
70 & 0.782 \\
71 & 0.638 \\
\hline
\end{tabular}

\section{ARTEFACTS IN THE GEOGRAPHICAL PATTERNS OF INCIDENCE}

Fig. 3 illustrates the association between standard incidence ratios for prostate cancer and the percentage of local tumours at county level.

The plot shows a clear positive trend amongst 18 of the counties (i.e. as expected higher percentage of local tumours are associated with higher incidence). However, Finnmark is an outlier with very low incidence and the highest $\%$ of local tumours. Because of this outlier the simple association is not statistically significant $(p=0.523)$ but when Finnmark is excluded the relationship amongst the remaining 18 counties is positive and highly statistically significant $(\mathrm{p}=0.011)$.

Pursuing the same association at the level of communes, using Poisson regression and averages of $\%$ local by hospital districts produces a highly significant $(\mathrm{p}<0.001)$ positive association:

$\log$ SIR $=-0.7766+0.01153 \%$ local

(standard error 0.0017)

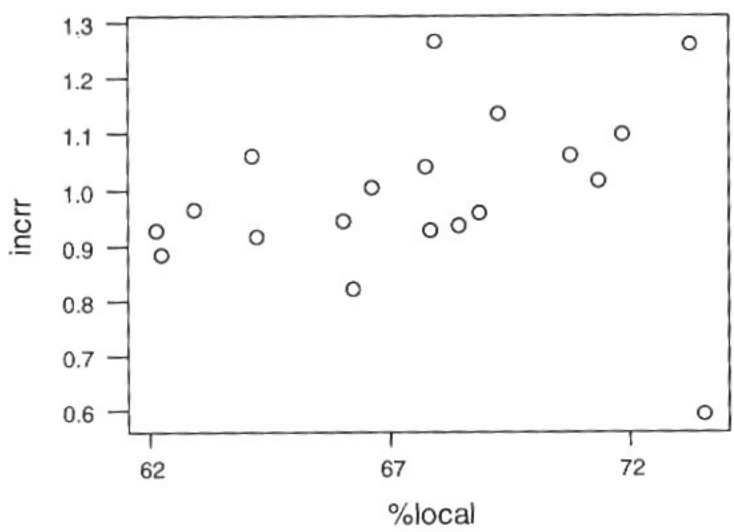

Figure 3. The association between standard incidence ratios for prostate cancer and the percentage of local tumours at county level in Norway, 1982-91.
This result was then used to adjust the expected value in each commune to take into account the tendency for incidence to be higher in areas with high \% local tumours (i.e. adjusting for potential artefacts in the SIR). Observed and adjusted expected values were then aggregated into counties and the results are shown in Table 2, with unadjusted SIRs shown for comparison.

Table 2. Standard incidence ratios for counties, before and after adjusting for $\%$ local cancers.

\begin{tabular}{lcc}
\hline County & unadjusted SIR & adjusted SIR \\
\hline Østfold & 0.937 & 0.986 \\
Akershus & 0.966 & 1.018 \\
Oslo & 1.015 & 0.970 \\
Hedmark & 1.005 & 1.013 \\
Oppland & 0.915 & 0.949 \\
Buskerud & 0.885 & 0.941 \\
Vestfold & 0.958 & 0.942 \\
Telemark & 0.936 & 0.924 \\
Aust-Agder & 1.261 & 1.181 \\
Vest-Agder & 1.062 & 1.022 \\
Rogaland & 1.267 & 1.225 \\
Hordaland & 1.042 & 1.037 \\
Sogn og Fjordane & 1.135 & 1.111 \\
Møre og Romsdal & 0.946 & 0.960 \\
Sør-Trøndelag & 1.099 & 1.044 \\
Nord-Trøndelag & 1.061 & 1.102 \\
Nordland & 0.927 & 0.922 \\
Troms & 0.821 & 0.832 \\
Finnmark & 0.593 & 0.554 \\
\hline
\end{tabular}

It is clear that this adjustment has some effect but the major features of the geographical pattern of incidence remain similar to those shown by the original unadjusted SIRs. It is particularly noteworthy that the very low incidence in Finnmark is actually lower after adjustment, which means that it has a very low SIR even though it has a high \% of local tumours which is generally associated with a tendency towards high incidence.

In fig. 4 the map shows variations in incidence of prostate cancer for counties after adjustment for $\%$ local tumours. The pattern is largely similar to the one for unadjusted SIRs as shown in fig. 1 .

Fig. 5 shows the significant deviations in SIRs after adjustment for communes. Compared with the pattern of unajusted SIRs (fig. 2), the number of significantly negative deviations for Finnmark has increased from six to nine, whereas many of the units in the rest of the country have disappeared. The significantly positive deviations remain with the same number and largely the same pattern.

Further evidence of artefacts in the incidence data can be demonstrated by calculating the ratio of SIR to standard mortality ratio (SMR). The hypothesis is that 


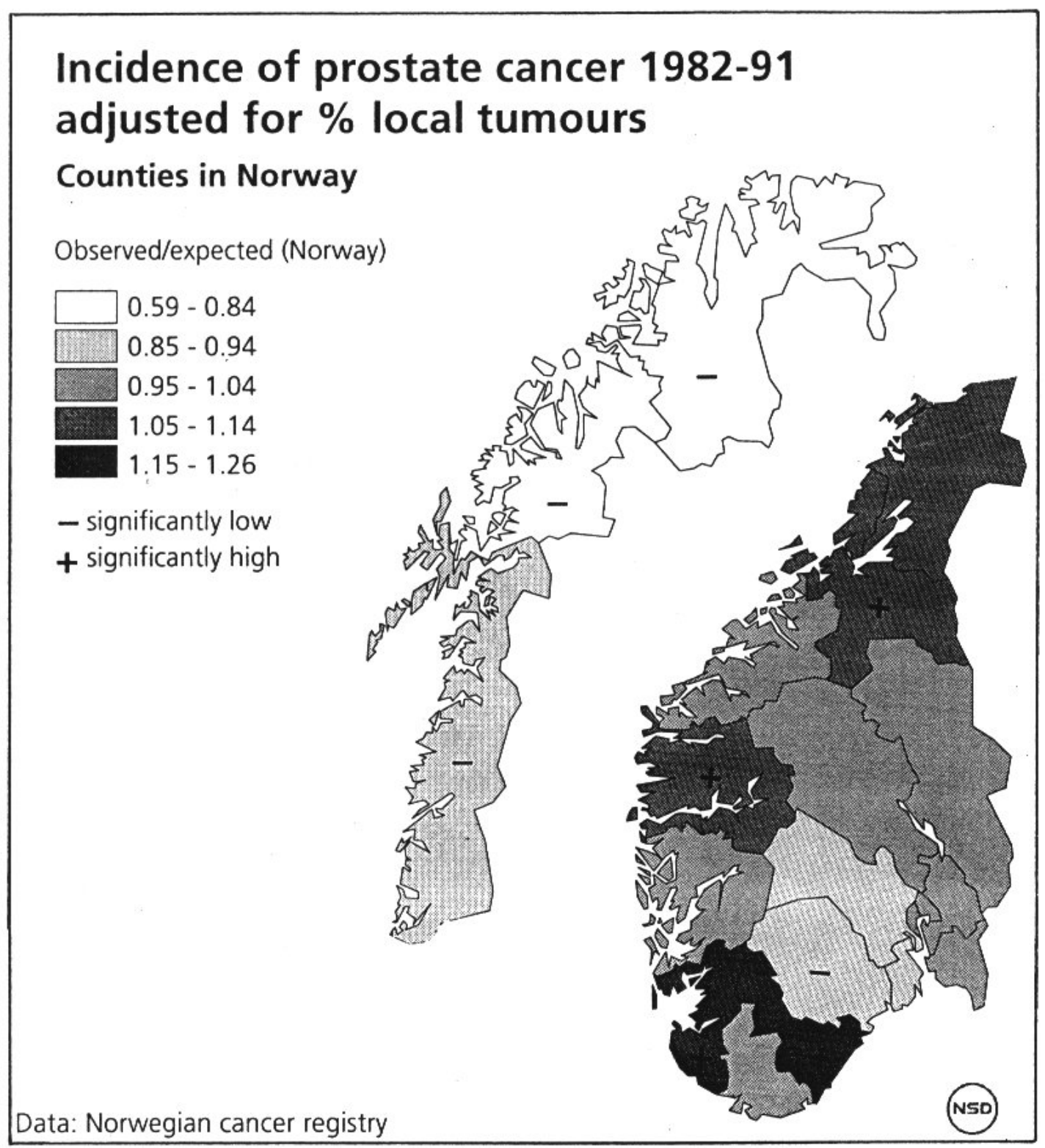

Figure 4. Incidence of prostate cancer adjusted for \% local tumours in Norwegian counties, 1982-91.

this ratio will be higher where the $\%$ of local tumours is high. This could be because the high \% of local tumours is indicative of health care activity leading to a more complete registration of tumours at earlier stages and these are less likely to be fatal. Figure 6 shows a highly significant $(\mathrm{p}<0.001)$ positive association which supports this hypothesis.

\section{CONCLUSION}

Some of the geographical variations in prostate cancer incidence in Norway may be artefactual due to variations in the intensity of activities likely to lead to the diagnosis of latent tumours. However, there also appear to be substantial real differences in incidence

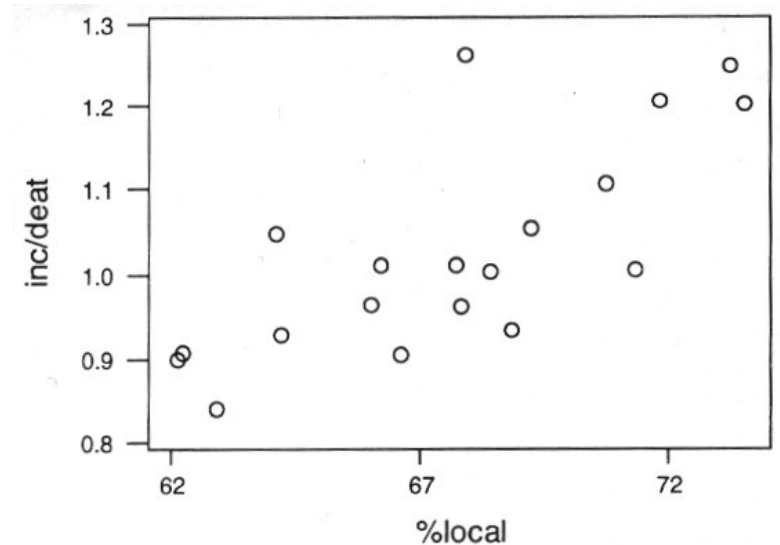

Figure 6. The association between \% local tumours and the ratio of SIR to SMR at county level in Norway, 1982-91. 
between different areas. The most striking trend is for the zone of low rates in the far north to remain, and even intensify, after the adjustment for \% local tumours has been made. There now seems to be a basis for using adjusted incidence data as well as mortality data in the search for etiological clues of the causes of geographical variations in prostate cancer in Norway.

\section{ACKNOWLEDGEMENTS}

Thanks to Dr. Sverre Harvei of the Norwegian Cancer Registry for assistance with interpreting the incidence data and valuable comments on our manuscript. The responsibility for the choice of approach and the conclusions is still with the two authors. Thanks also to the Norwegian Social Science Data Service for producing the maps.

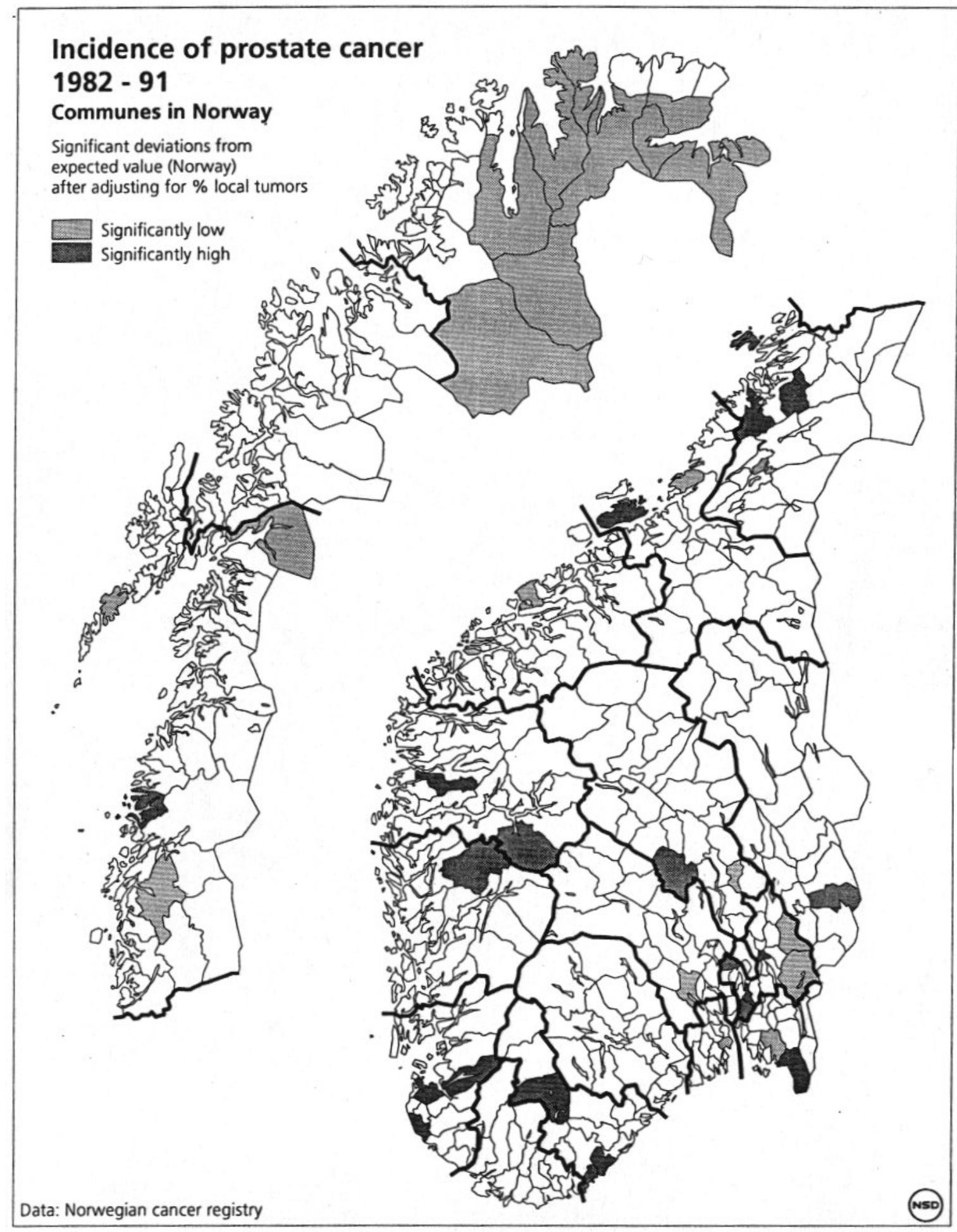

Figure 5. Incidence of prostate cancer adjusted for \% local tumours in Norwegian communes, 1982-91. 


\section{REFERENCES}

Boyle P, Maisonneuve P, Napalkov P. Geographic and temporal patterns of incidence and mortality from prostate cancer. Urology 1995; 46: 47-55.

Gilliland FD, Welsh DJ, Hoffmann RM, Key CR. Rapid rise and subsequent decline in prostate cancer incidence rates for New Mexico, 1989-1993. Cancer Epidemiol Biomark Prevent 1995; 4: 797-800.

Gilliland FD, Hunt WC, Key CR. Ethnic variation in prostate cancer survival in New Mexico. Cancer Epidemiol Biomark Prevent 1996; 5: 247-251.

Grönberg H, Bergh A, Damber J-E, Jonsson H, Lenner P, Ångström T. Prostate cancer in northern Sweden. Incidence, survival and mortality in relation to tumour grade. Acta Oncol 1994; 33 (4): 359-363.

Grönberg H, Damber L, Damber J-E. Total food consumption and body mass index in relation to prostate cancer risk: A case-control study in Sweden with prospectively collected exposure data. J Urol 1996; 155: 969-974.

Harvei S, Tretli S, Langmark F. Cancer of the prostate in Norway 1957-1991 - A descriptive study. Eur J Cancer 1996; 32A (1): 111-117.

Helgesen F, Holmberg L, Johansson J-E, Bergström R, Adami H-O. Trends in cancer survival in Sweden, 1960 through 1988: Evidence of increasing diagnosis of non-lethal tumours. J Natl Cancer Inst 1996; 88 (17): 12161221.

Jacobson SJ, Katusic SK, Bergstralh EJ, Oesterling JE, Ohrt D, Klee GG, Chute CG, Lieber MM. Incidence of prostate cancer diagnosis in the eras before and after serum prostate-specific antigen testing. JAMA 1995; 274: 1445-1449.

Key T. Risk factors for prostate cancer. Cancer Surv 1995; 23: 63-76.

Kreftregisteret. Forekomst av kreftsykdommer i Norges kommuner. Antall nye tilfeller 1982-1991. Antall personer med tidligere kreftdiagnose i live pr. 1.1. 1992. Oslo: Kreftregisteret, 1993.

Muir CS, Nectoux J, Staszewski J. The epidemiology of prostate cancer. Geographical distribution and timetrends. Acta Oncol 1991; 30 (2): 133-140.

Pedersen PB, Jørgensen S (eds). Samdata - Sykehus 1990. Sammenlikningsdata for somatiske sykehus. SINTEF/NIS-rapport 1/91. Trondheim: Norsk institutt for sykehusforskning (Norwegian Institute for Hospital Research), 1991.

Ross RK, Schottenfeld D. Prostate cancer. In: Schottenfeld D, Fraumeni JF (eds.) Cancer epidemiology and prevention. Oxford: Oxford University Press, 1996: 1180-1206. 